\title{
Modeling T cell receptor recognition of CD1-lipid and MR1-metabolite complexes
}

\author{
Brian G Pierce ${ }^{1,2^{*}}$, Thom Vreven ${ }^{1}$ and Zhiping Weng ${ }^{1 *}$
}

\begin{abstract}
Background: T cell receptors (TCRs) can recognize diverse lipid and metabolite antigens presented by MHC-like molecules CD1 and MR1, and the molecular basis of many of these interactions has not been determined. Here we applied our protein docking algorithm TCRFlexDock, previously developed to perform docking of TCRs to peptide-MHC (pMHC) molecules, to predict the binding of $a \beta$ and $\gamma \delta$ TCRs to CD1 and MR1, starting with the structures of the unbound molecules.
\end{abstract}

Results: Evaluating against TCR-CD1d complexes with crystal structures, we achieved near-native structures in the top 20 models for two out of four cases, and an acceptable-rated prediction for a third case. We also predicted the structure of an interaction between a MAIT TCR and MR1-antigen that has not been structurally characterized, yielding a top-ranked model that agreed remarkably with a characterized TCR-MR1-antigen structure that has a nearly identical TCR a chain but a different $\beta$ chain, highlighting the likely dominance of the conserved a chain in MR1-antigen recognition. Docking performance was improved by re-training our scoring function with a set of TCR-pMHC complexes, and for a case with an outlier binding mode, we found that alternative docking start positions improved predictive accuracy. We then performed unbound docking with two mycolyl-lipid specific TCRs that recognize lipid-bound CD1b, which represent a class of interactions that is not structurally characterized. Highly-ranked models of these complexes showed remarkable agreement between their binding topologies, as expected based on their shared germline sequences, while differences in residue-level interactions with their respective antigens point to possible mechanisms underlying their distinct specificities.

Conclusions: Together these results indicate that flexible docking simulations can provide accurate models and atomic-level insights into TCR recognition of MHC-like molecules presenting lipid and other small molecule antigens.

Keywords: TCR, MAIT, CD1d, CD1b, GEM, MHC-like

\section{Background}

$\mathrm{T}$ cell receptors (TCRs) display remarkable versatility in their ability to specifically recognize a wide array of structurally and chemically diverse antigens. This is highlighted by a number of studies showing that, in addition to well-characterized recognition of peptide-major histocompatibility complex (pMHC) [1], many TCRs engage MHC-like molecules CD1, which present a variety of lipid antigens [2], and MR1, which presents vitamin B metabolites [3]. At the sequence level, these TCRs can be restricted or diverse in their germ-line chain usage,

\footnotetext{
*Correspondence: pierce@umd.edu; zhiping.weng@umassmed.edu ${ }^{1}$ Program in Bioinformatics and Integrative Biology, University of Massachusetts Medical School, 368 Plantation Street, Worcester, MA 01605, USA

2University of Maryland Institute for Bioscience and Biotechnology Research, 9600 Gudelsky Drive, Rockville, MD 20850, USA
}

depending in part on the $\mathrm{T}$ cell type, antigen, and antigen-presenting molecule [2].

The understanding of the molecular basis of TCR interactions with CD1 and MR1 has been greatly advanced by a number of crystallographic studies that have elucidated the interface sites, key contacts, and binding modes of several of these complexes. This includes type I and type II natural killer T cell (NKT) TCR interactions with CD1d-lipid [4-6], mucosal-associated invariant $\mathrm{T}$ cell (MAIT) TCR interfaces with unliganded [7] and ligand-bound MR1 [8], and recent studies of two $\gamma \delta$ TCRs bound to CD1d-lipid [9,10]. While these have provided a clear view of the antigen recognition underlying several of the invariant and diverse $T$ cell subsets, owing to their variety and the effort required in experimental 
structure determination, the crystal structures of a number of key complexes have not yet been solved.

Here we describe the adaptation of the TCR docking algorithm, TCRFlexDock, previously shown to produce highly accurate TCR-pMHC structural predictions [11], to predict complexes of TCRs with CD1 and MR1 with antigen (Ag). We assessed unbound docking performance using four known structures of TCRs with CD1d, showing that for most cases accurate models were produced. We also predicted the complex between a MAIT TCR and MR1-Ag, an interaction that has not yet been structurally described, and assessing models against a related MAIT TCR bound to MR1-Ag, found strong agreement between predicted complex and the likely conserved binding mode. Applying TCRFlexDock to predict the structure of two germline-encoded, mycolyl lipid-reactive (GEM) TCRs bound to CD1b, we produced models that provide insight into both their shared sequence features and distinct antigen specificities.

\section{Results}

Prediction of TCR-CD1d and TCR-MR1 complexes

Searching for existing unbound and bound TCR and MHC-like protein structures in the Protein Data Bank (PDB) [12], we identified four TCR-CD1d-Ag test cases (Table 1) representing various classes of TCRs (Type I NKT TCR, Type II NKT TCR, and two $\gamma \delta$ TCRs). Additional complexes of the same class with essentially identical docking orientations (e.g. Type I NKT TCRs bound to CD1d and other antigens) were not included. We also identified a case with an unbound MAIT TCR (TRAV1-2TRAJ33-TRBV20 germ line) that binds MR1 presenting the antigen 6FP (6-formyl pterin, a vitamin B metabolite), with a nearly identical sequence to the $\alpha$ chain from a structure of another MAIT TCR (TRAV1-2-TRAJ33TRBV6 germ line) bound to MR1-6-FP (Table 1); two residues are substituted near the N-term of the CDR3 $\alpha$ loop. Given the likely "common mode of MAIT TCR-MR1 docking" [13], supported by additional MAIT TCR complexes with bovine MR1 and TRBV6 TCR variants [14], we evaluated docking predictions of the unbound TRBV20
MAIT TCR to MR1-6FP using the conserved $\alpha$ chain and MR1 in the bound structure, to determine whether the $\alpha$ chain binding conformation is recapitulated by docking, and to predict how the distinctive TRBV20 chain engages MR1-Ag. Collectively, these TCR-CD1d-Ag and TCR-MR1Ag structures represent a wide variety of docking modes (Figure 1), with greater variability than the TCR-pMHC complexes we considered in our previous work [11].

We performed flexible protein docking using TCRFlexDock to predict the complexes in Table 1 using the unbound TCR and CD1d-Ag/MR1-Ag structures; results are shown in Table 2, with test cases represented by their complex PDB IDs from Table 1. Scores versus interface root-mean-square distances (RMSDs) for cases with hits are shown in Figure 2. We assessed models using CAPRI criteria [21], classifying them as incorrect, acceptable, medium accuracy, or high accuracy, and defined hits, as in our previous study of TCR-pMHC docking [11], as those with medium or high accuracy. For two out of the four CD1d test cases in Table 1 (4EI5 and 4LHU), hits were identified in the top 20 predictions, ranked as in our previous work by ZRANK 2 (ZR2). For test case 4 MNG $(\gamma \delta$ TCR-CD1d-sulfatide), relatively few hit predictions were generated, and these were not well-ranked (the top hit was ranked 235). Despite its rigid-body classification due to minimal binding conformational changes, this case was challenging due to its atypical docking orientation featuring only the $\delta$ TCR chain contacting CD1d-sulfatide [10], resulting in a significant distance from the docking start position as well as a smaller interface area which is unfavorable for the docking scoring function. However, nearhits (acceptable predictions; in parentheses in Table 1) were highly ranked for this case, with a top rank of 10 . For test case 3HUJ, no hits were found among the 1000 TCRFlexDock models, which was likely due to its outlier binding mode and distance from the starting docking orientation (over $20 \AA$ ligand RMSD). Performance in this case was improved by employing alternative docking start sites closer to its bound orientation, as noted below.

For the MAIT TCR-MR1-6FP case, the predictions were remarkably consistent with a conserved MAIT TCR $\alpha$

Table 1 The TCR-CD1d-Ag and TCR-MR1-Ag test cases

\begin{tabular}{|c|c|c|c|c|c|c|c|}
\hline \multicolumn{3}{|c|}{ PDB code } & \multirow[b]{2}{*}{ TCR name } & \multirow[b]{2}{*}{ TCR type } & \multirow[b]{2}{*}{ Ligand name } & \multirow[b]{2}{*}{ Binding RMSD ${ }^{1}, \AA$} & \multirow[b]{2}{*}{ Difficulty $^{2}$} \\
\hline Complex & TCR & Ligand & & & & & \\
\hline $3 \mathrm{HUJ}$ [15] & 2 EYS [16] & 1ZT4 [17] & NKT15 & Type I NKT & CD1d-a-GalCer & 1.04 & Rigid \\
\hline $4 \mathrm{EI} 5[5]$ & $4 \mathrm{El6}[5]$ & 2AKR [18] & XV19 & Type II NKT & CD1d-sulfatide & 1.08 & Medium \\
\hline 4LHU [9] & 4LFH [9] & 1ZT4 [17] & $9 \mathrm{C} 2$ & $\gamma \delta$ & CD1d-a-GalCer & 0.90 & Rigid \\
\hline $4 \mathrm{MNG}[10]$ & $4 \mathrm{MNH}[10]$ & 4MQ7 [10] & DP10.7 & $\gamma \delta$ & CD1d-sulfatide & 0.68 & Rigid \\
\hline $4 \mathrm{~L} 4 \mathrm{~T}[8]^{3}$ & 4DZB $[19]^{3}$ & 4GUP [3] & MAIT & MAIT & MR1-6FP & 0.87 & Rigid \\
\hline
\end{tabular}

${ }^{1}$ Backbone atom root-mean-square distance between interface residues in the bound structure and corresponding residues in the unbound structures.

${ }^{2}$ Docking difficulty, based on extent of binding conformational changes [20].

${ }^{3}$ The bound and unbound MAIT TCRs are closely related, with nearly identical a chains; the differing $\beta$ chains (TRBV6 in 4L4T and TRBV20 in 4DZB) were excluded from RMSD calculations and evaluation of docking predictions. 


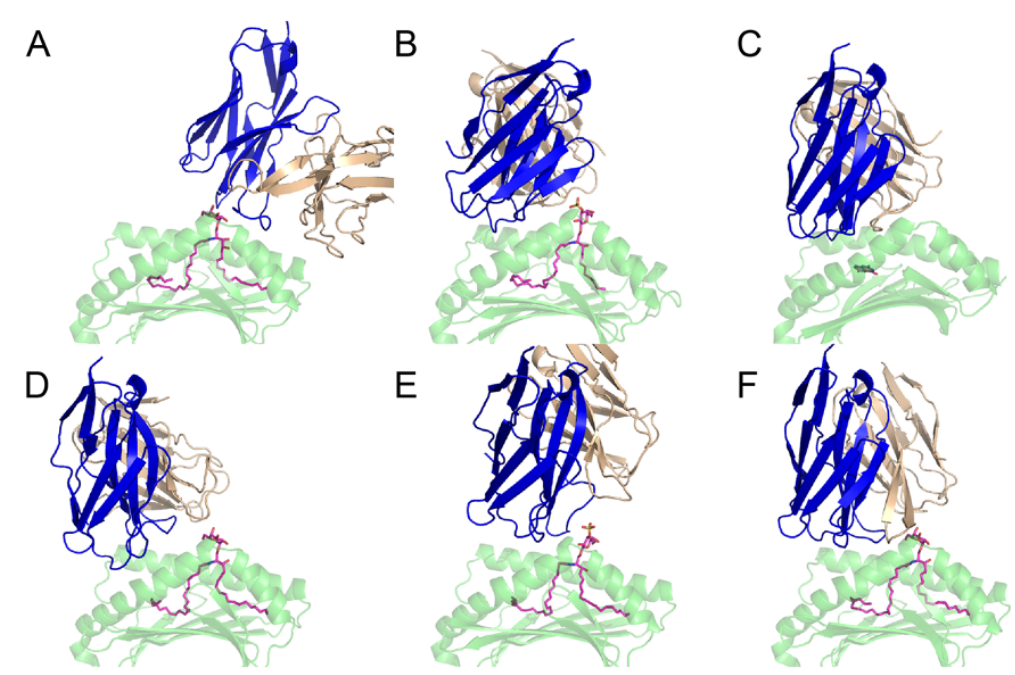

Figure 1 The structures and starting position of the docking test cases. Bound structures of (A) 3HUJ, (B) 4EI5, (C) 4L4T, (D) 4LHU, (E) 4MNG, as well as a representative docking start position (F) are shown (test case $3 \mathrm{HUJ}$ ), with TCRs colored blue (a chain) and gold ( $\beta$ chain), CD1 and MR1 green, and antigens magenta. Not shown are TCR constant domains, as well as the a3 domain and $\beta 2 \mathrm{~m}$ of CD1d and MR1.

chain binding conformation, with the top-ranked model involving the TRBV20 TCR classified a hit with respect to the bound crystal structure with the TRBV6 TCR, and the third-ranked model having the lowest RMSD $(1.07 \AA)$ from the bound structure among all docking models. This model, shown in Figure 3, exhibits nearly perfect matches with TCR $\alpha$ backbone and key side chains in the crystal structure (e.g. hot spot TCR residue Y95 $\alpha$ ). In contrast, the modeled TRBV20 and crystal structure TRBV6 chains differ somewhat in CDR loop structure and overall orientation. This is consistent with CDR3 $\beta$ swapping experiments between the TRBV20 and TRBV6 MAIT TCRs considered here that ablated MR1-Ag binding, which implied a TRBV germline context dependence for CDR3 $\beta$ loops ("possibly via steric hindrance mechanisms") [19]. Furthermore there are relatively few favorable side chain contacts between modeled TRBV20 $\beta$ chain and MR1, in agreement with alanine scanning mutagenesis data [19] that found no individual $\beta$ chain CDR mutants of this MAIT TCR that significantly altered MR1 recognition.
To investigate whether the TCR CDR loop backbone structure had a significant relationship with overall docking accuracy for these cases, we compared RMSD for modeled versus bound TCR residue backbone atoms (for the subset of TCR residues within $10 \AA$ of $\mathrm{CD} 1-\mathrm{Ag}$ ) with rigid-body ligand RMSD for the 1000 docking models of two test cases (Additional file 1: Figure S1). Surprisingly, there was little relationship between these values, indicating that although a bound-like TCR backbone will improve the hit rate of the docking simulation as we previously noted [11], the sampling of CDR loop conformations during docking includes bound-like conformations for both hits and non-hits, and a near-native docking position (i.e. low ligand RMSD) does not necessarily lead to accurately-modeled CDR loops.

\section{Retraining the TCRFlexDock scoring function}

We re-trained the ZRANK scoring function using the previously reported set of TCR-pMHC test cases and docking results [11] to determine whether such an

Table 2 The predictive docking performance starting from the "start1" site

\begin{tabular}{|c|c|c|c|c|c|c|}
\hline \multirow[b]{2}{*}{ Test case } & \multicolumn{2}{|c|}{ Start RMSD, Å } & \multirow[b]{2}{*}{ Hits $^{1}$} & \multirow[b]{2}{*}{ ZR2 Rank ${ }^{2}$} & \multirow[b]{2}{*}{ ZRT Rank ${ }^{2}$} & \multirow[b]{2}{*}{ T20 RMSD ${ }^{3}, \AA$} \\
\hline & Ligand & Interface & & & & \\
\hline $3 \mathrm{HUJ}$ & 21.23 & 9.67 & 0 & - & - & 7.91 \\
\hline $4 \mathrm{El} 5$ & 8.95 & 3.43 & 7 & 6 & 1 & 2.21 \\
\hline $4 \mathrm{LHU}$ & 8.22 & 2.42 & 45 & 17 & 8 & 1.97 \\
\hline $4 M N G$ & 13.9 & 5.06 & 6 & $235(10)$ & $107(8)$ & 3.22 \\
\hline $4 \mathrm{~L} 4 \mathrm{~T}^{4}$ & 5.77 & 2.63 & 38 & 1 & 1 & 1.07 \\
\hline
\end{tabular}

${ }^{1}$ Number of hit predictions among the 1000 models from TCRFlexDock.

${ }^{2}$ Rank of the first hit; for 4 MNG, values in parentheses denote the ranks of the first "acceptable" prediction.

${ }^{3}$ Lowest interface root mean square distance from bound in the top 20 predictions, ranked by the ZRT scoring function.

${ }^{4}$ Only TCR a chain and MR1 were used to evaluate these predictions, as unbound and bound TCR $\beta$ chains differ in sequence. 

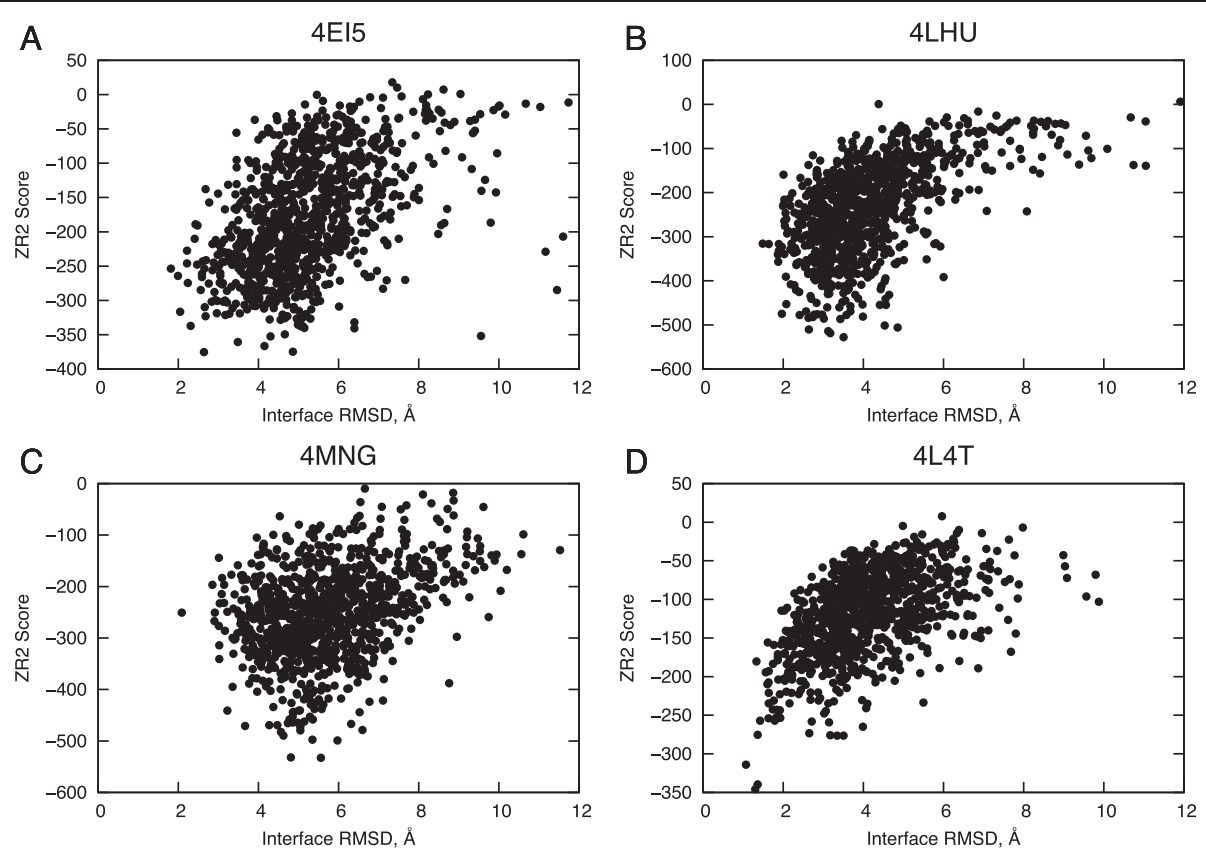

Figure 2 Binding funnels of TCR-CD1d and TCR-MR1 test cases. Scores from ZRANK 2 (ZR2) versus interface RMSDs are given for (A) 4EI5, (B) $4 \mathrm{LHU}$, (C) $4 M N G$, and (D) $4 L 4 T$, with only residues from the a chain and MAIT being used to calculate interface RMSD for (D).

optimized function would improve success on the TCRCD1 and TCR-MR1 cases in Table 1 . The re-weighted function we derived (named ZRT, for ZRANK TCR) indeed led to improved predictive performance in Table 2; though the first hit for case 4MNG was still ranked relatively low, its rank improved (rank 107, versus 235 for ZR2), while the near-hit was ranked 8 by ZRT (versus 10 by ZR2). The new TCR-pMHC derived weights had a lower van der Waals attractive weight relative to the other terms compared with the previous ZRANK function [22], likely due to the lower shape complementarity of TCRpMHC interactions versus protein-protein interfaces in general [23]. The top-ranked ZRT prediction for test case 4EI5 had a $2.21 \AA$ interface RMSD from native; its structure is shown in Additional file 2: Figure S2. Despite some deviations of the sulfatide antigen head group and CDR3 $\beta$ with respect to the complex crystal structure, the XV19 TCR variable domains as a whole, as well as several key interface side chains, are positioned similarly to those of the known complex.

\section{Utilizing alternate docking start sites}

Considering the distinct binding orientation of the Type I NKT TCR test case 3HUJ (Figure 1), we initialized separate docking simulations, in addition to the original "start1" docking start site, from two alternative sites closer to the bound orientation to determine whether they would lead to TCRFlexDock hits. We employed a "start2" site that was approximately equidistant from the 3HUJ and 4MNG bound orientations, with a $20 \AA$ translational shift along the CD1d helices from "start1" (see Methods), as well as a "fit" site that entailed root-mean-

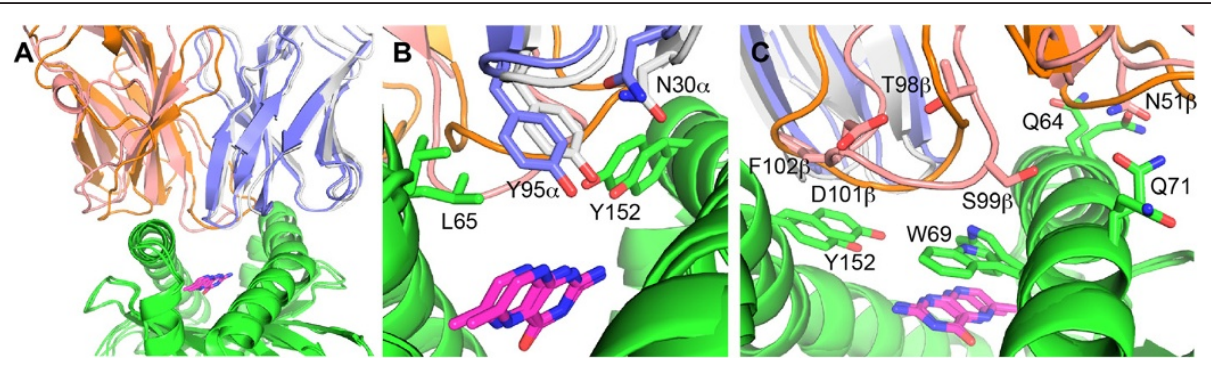

Figure 3 Predictions of the MAIT TCR-MR1 complex compared with crystal structure of complex with related MAIT TCR. Shown are the (A) complex, (B) close-up of the a chain interface with MR1-Ag, and (C) close-up of the $\beta$ chain interface with MR1-Ag, with selected residues show as sticks. Predicted TCR is blue (a chain) and salmon ( $\beta$ chain), TCR from crystal structure (PDB code 4L4T) is gray (a chain) and orange ( $\beta$ chain), MR1 is green, and 6-formyl pterin antigen is magenta. Structures were superposed by MR1 helices. 
square fitting the unbound TCR to the bound TCR position (Figure 4). Despite substantial distance from the bound orientation (13.65 A ligand RMSD), docking from the start 2 site led to highly ranked hits using both ZR2 and ZRT scoring functions for 3HUJ (top hits ranked 10 and 8, respectively; Additional file 3: Table S1), though some non-hits from start1 docking ranked higher than these hits (Figure 4). Hits were not improved for test case $4 \mathrm{MNG}$, indicating as noted above that the interface characteristics rather than the docking orientation alone likely caused the docking search to yield few favorable hits. As expected from their relatively large distances to the bound conformation ( $>18 \AA$ ligand RMSD), for the remaining three cases the start 2 docking start site led to no hits (Additional file 3: Table S1). When pooling results from start1 and start2 sites however, the nearnative predictions were predominantly scored better by ZRT for all these cases (Additional file 4: Figure S3). For one test case (4LHU) we also tested pooling results from two additional start sites (one intermediate site between start1 and start2, and an extreme start site past start2), and ZRT continued to rank the near-native models best (Additional file 5: Figure S4).

\section{Prediction of TCR complexes with CD1b-GMM and CD1 b-MA}

Recently the unbound crystal structures of two clones of germline mycolyl-lipid reactive (GEM) $\alpha \beta$ TCRs were reported [24]: clone 18 (PDB code 4G8E), which binds CD1b-MA, and clone 42 (PDB code 4G8F) which binds CD1b-GMM. As the complex structures have not been reported, and the structure of CD1b-GMM was reported in a previous study [25], we used TCRFlexDock to predict these two structures representing this class of interactions. As the $\alpha$ chains are nearly identical between the two GEM TCR clones and likely dominate the interactions with CD1b-Ag [24], we computed the distances between the models ranked in the top 20 by ZRT for the two TCRs based on the RMSDs between their germline $\mathrm{CDR} \alpha$ loop positions (with CD1b superposed; Additional file 6: Figure S5). We found that the 4G8E and 4G8F models (for brevity, we refer to unbound TCR PDB codes to represent these two complexes) were highly similar to each other, with many of the top 20 models less than $4 \AA$ apart. 4G8E model 3 and 4G8F model 12 exhibited the highest similarity in germ line $\operatorname{CDR} \alpha$ positioning (1.37 $\AA$ RMSD) over CD1b-Ag, and fell into the largest cluster of models in both sets of top 20 predictions (the bottom left cluster in Additional file 7: Figure S6). On this basis, as well as notable contacts with antigen as described below, we selected these models for further analysis.

The structures of these models (Figure 5) provide a view of their overall recognition mode as well as several differences in TCR contacts mediating differential antigen recognition. GEM TCR engagement of CD1b is similar to characterized structures of $\alpha \beta$ TCR recognition of pMHCs, including the murine Yae62 TCR bound to H-2 $\mathrm{K}^{\mathrm{b}}$ and peptide (Additional file 7: Figure S6 and Figure 5A). Closer examination of the modeled interfaces shows key roles for certain TCR residues in mediating antigen recognition, for instance Tyr31 $\beta$ is positioned close to the antigen in both complexes. Arg107 $\beta$ of the 4G8E TCR is of particular interest, due to its potential electrostatic

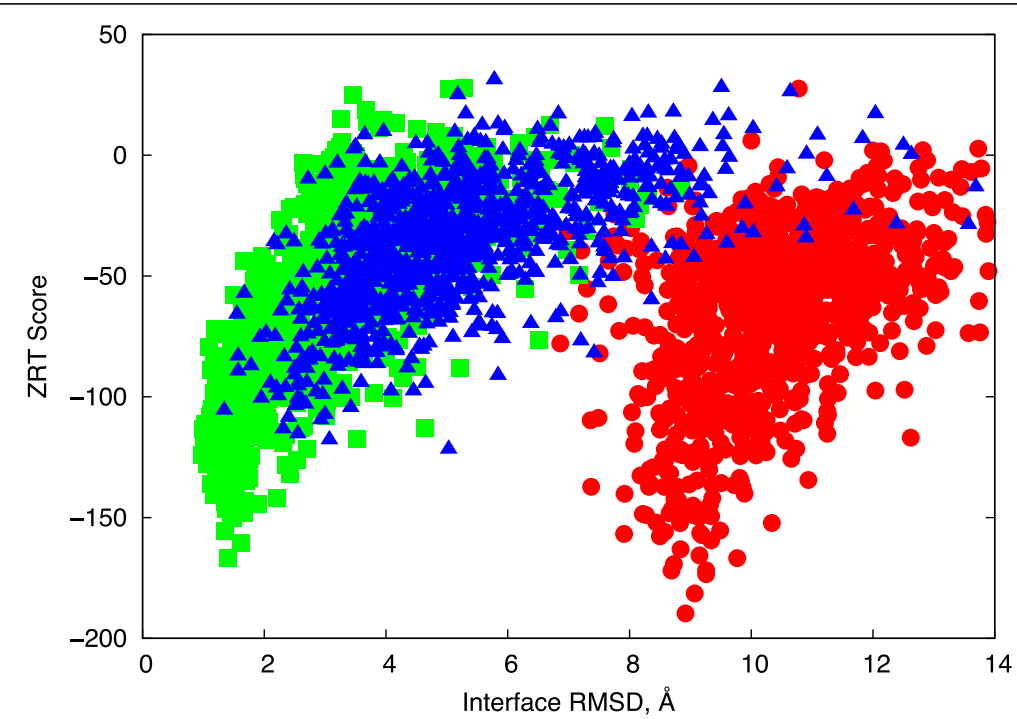

Figure 4 Binding funnels of the 3HUJ Type I NKT TCR test case. ZRANK TCR (ZRT) scores versus interface RMSDs are shown for TCRFlexDock simulations starting from the "start1" TCR start position (red circles), "fit" TCR start position (green squares), and shifted "start2" start position (blue triangles). 


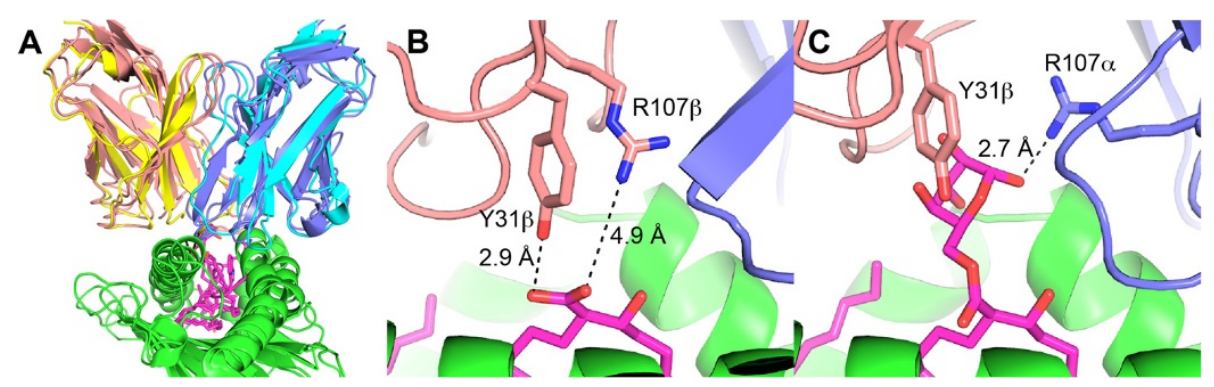

Figure 5 Predictions of GEM TCR complexes with CD1b-Antigen. (A) GEM TCR models bound to CD1b-Ag and Yae62 TCR bound to H-2 $K^{b}$-peptide (PDB code 3RGV). Colors are: GEM a chains, blue; GEM $\beta$ chains, salmon; Yae62 a chain, cyan; Yae62 $\beta$ chain, yellow; CD1b/MHC, green; antigens MA/GMM/peptide, magenta. (B) Clone 18 GEM TCR modeled interface with CD1b-MA. (C) Clone 42 GEM TCR modeled interface with CD1b-GMM. For (B) and (C), key TCR side chains contacting antigen head group and inter-atomic distances are indicated.

interaction with the negatively charged head group of the MA antigen (Figure 5B); this residue was also highlighted based on the unbound clone 18 structure as it represented a notable difference between the two clones' CDR3 architectures [24]. While these models provide a likely mechanism of CD1b-Ag engagement, other sets of $4 \mathrm{G} 8 \mathrm{E}$ and $4 \mathrm{G} 8 \mathrm{~F}$ models were identified that shared docking orientations with TCR-pMHC and MAITMR1-Ag interfaces (Additional file 7: Figure S6 and Additional file 8: Figures S7).

\section{Discussion}

We have demonstrated that our TCR docking algorithm, TCRFlexDock, is capable of producing accurate models of TCRs recognizing MHC-like molecules presenting a variety of non-peptide ligands. By retraining our scoring function, we were able to improve the re-ranking performance for test cases representing this class of complexes. Further improvements, for instance to improve the ranking of hits for the $4 \mathrm{MNG}$ or $3 \mathrm{HUJ}$ cases and performance using pooled results from alternate docking start sites, could potentially be achieved through inclusion of other terms (e.g. longrange partial charge rather than full charge electrostatics), and further optimization of the pair potential parameters and antigen atom typing. Additionally, though antigen bond torsion angles were minimized during Rosetta docking simulations, explicit inclusion of lipid head group rotamers (based on pre-generated conformers) could represent another route for improving docking performance.

We report models of CD1b-bound GEM TCRs from TCRFlexDock that account for a likely shared docking orientation as well fine differences in antigen recognition. The docking searches for these cases were focused on the CD1b site analogous to the pMHC interface engaged by most TCRs; while outlier TCR binding modes have been observed for some non-MHC ligands [26] and would reduce change of docking success (as seen for test case 3HUJ), mutagenesis evidence strongly suggests that TCRs engage CD1b-Ag with a footprint similar to TCR-
pMHC interactions [27]. Two previously reported TCRCD1b-Ag modeling attempts (using other TCR clones) were based on homology with TCR-pMHC complex structures [25,28], rather than a flexible docking search; notably, in one of these studies the TCR loops exhibited considerable clash with antigen in the modeled complexes [25], a challenge that is explicitly addressed during the conformational search of TCRFlexDock. Within our set of TCR-CD1b-Ag models, the dominant cluster was similar to the Class I MHC-bound Yae62 TCR (Figure 5 and Additional file 7: Figure S6). However, we did identify alternative models, including several that were similar to the TRBV6 MAIT TCR-MR1-Ag and ELS4 TCR-HLA-peptide complexes. As noted by others [24], the MAIT, ELS4 and GEM TCRs share TRAV1-2 genes and the former two exhibit similar docking orientations over their respective ligands [8], yet given the structural and residue-level differences between CD1b, MR1 and $\mathrm{MHC}$, there is a significant possibility that TCR-CD1b-Ag interactions exhibit a distinct binding conformation, as we identified using TCRFlexDock. Experimental structural characterization of GEM TCR-CD1b-Ag complexes will allow determination of which models are most accurate.

While this manuscript was under review, the $\mathrm{x}$-ray structure of a TRBV20-containing MAIT TCR (corresponding to the unbound TCR we used for test case 4L4T) in complex with MR1 and antigen was reported [29]. Though the antigens of our docking simulation and the solved structure are not identical (thus preventing an analysis of native contacts and hits), we evaluated interface backbone RMSD between our models and the solved structure (PDB id 4PJ8), and the top-ranked model by ZRT score indeed had a low interface RMSD with respect to the experimentally determined complex structure (1.68 Å; Additional file 9: Figure S8).

\section{Conclusions}

These updates to the TCRFlexDock algorithm, as well as the docking test cases we assembled, represent an advance 
in modeling TCR-ligand recognition, extending capabilities beyond TCR-pMHC modeling methods reported by ourselves [11] and others [30,31] to modeling complexes with lipid and metabolite antigens presented by MHC-like molecules. Our models of MAIT TCR-MR1-Ag and GEM TCR-CD1b-Ag complexes, which have not been described crystallographically, provide likely mechanisms of ligand engagement by these TCRs, and highlight the ability of advanced protein docking algorithms to complement experimental techniques in probing the structural basis of molecular recognition. These methods can be applied to model other TCRs bound to MHC-like ligands, as more of these interactions and TCRs become characterized, such as a $\gamma \delta$ TCR clone that was recently found to engage the endothelial protein $\mathrm{C}$ receptor [32].

\section{Methods}

\section{Input structures and TCR docking}

TCR docking test cases were identified based on identical sequences between TCRs and ligands from unbound and bound PDB entries. Lipid and metabolite antigens were also evaluated for unbound and bound structural matches. For test case 4LHU, the lipid molecules were not identical (JLS for the bound CD1d- $\alpha$-GalCer, AGH for the unbound CD1d- $\alpha-G a l C e r)$ but this was due to differences in buried hydrocarbon chains, while the exposed head groups were the same. Prior to docking, missing unbound TCR CDR loop residues were added to PDB files 4LFH (test case 4LHU), 4MNH (test case 4MNG), 4G8E, and 4G8F using Modeller [33], keeping the remainder of the TCR fixed. The unbound CD1b-MA structure was generated by truncation of the GMM antigen in the unbound CD1b-GMM structure (PDB code 1UQS).

Flexible TCR docking was performed in the same manner as presented previously [11], using a modified version of RosettaDock to generate 1000 docking models starting with unbound structures in a predetermined starting orientation (described in more detail below), followed by re-scoring docking models using ZRANK. RosettaDock simulations were performed using an iterative procedure (written with RosettaScripts) where the CDR3 loops undergo a perturbation using the kinetic closure (KIC) loop modeling protocol in Rosetta, followed by Monte Carlo-based sampling of local rigid-body docking orientation, side chain rotamer optimization, and two rounds of KIC refinement of all CDR loops. Parameters for lipid and antigens were generated using Open Babel [34] to convert from PDB to Mol2 format, followed by conversion to Rosetta parameter files using the molfile_to_params.py script [35]. This process was also used to determine antigen torsion angles that were minimized by Rosetta during the docking process. Docking was performed on a Linux cluster with $2.8 \mathrm{GHz}$ AMD Opteron cores; producing 50 docking models on a single core ( 20 such jobs were run in parallel for each test case) took approximately 10 hours on average (12 minutes per model). For ZRANK, antigen partial charge parameters were obtained from polar hydrogen Mol2 files from Open Babel, while IFACE and $\mathrm{ACE}$ atom types were assigned based on congruence with amino acid atoms.

\section{Initial and alternate docking input positioning}

As with our previous TCR docking study, for initial docking start position (corresponding to the "start1" site), TCRs were aligned with pseudo-symmetric axis perpendicular to the plane of the MHC-like helices, positioned over the MHC-like helix centroid at a distance of $25 \AA$, with a $45^{\circ}$ crossing angle. We also tested an alternate "start2" docking start site; for this site, with respect to "start1", the TCR was shifted by $20 \AA$ along the MHC-like helix axis toward the $\alpha 1$ helix C-term, rotated to a $0^{\circ}$ crossing angle, and tilted by $25^{\circ}$ toward the MHC-like helix axis C-term. Additional "start3" and "start4" sites included $10 \AA$ and $25 \AA$ TCR shifts, crossing angles of $22.5^{\circ}$ and $-15^{\circ}$, and tilts of $12.5^{\circ}$ and $40^{\circ}$, respectively.

\section{Evaluation of docking models}

Docking models were evaluated using CAPRI criteria to assess predictions as "incorrect", "acceptable", "medium accuracy" and "high accuracy" [21]. As with our previous TCR docking study, models assessed as medium or high accuracy are referred to as hits. Lipid and metabolite antigen atoms were included in calculation of bound contacts with TCR residues, but were omitted from RMSD calculations (only protein backbone atoms were used).

\section{Retraining ZRANK scoring function}

As for the original ZRANK implementation [36], we employed a downhill simplex to select weights for the terms of the ZRANK scoring function, in this case maximizing the average area under the receiver operating characteristic curve (AUC) of 20 TCR-pMHC docking test cases [11], distinguishing hit from non-hit predictions among the top 30 ranked models for each TCRpMHC case. To avoid local minima, the downhill simplex was run from 12,000 randomly generated starting points. For three-fold cross-validation within the TCRpMHC set, training and testing sets were selected such that cases with the same TCR were not used simultaneously in both sets. The retrained scoring function weights (and ZR2 weight values [22] in parentheses, for comparison) used to select TCR docking models are:

van der Waals attractive: $0.027(1.0)$

van der Waals repulsive: $1.33(0.23)$

Electrostatics short-range attractive: $0.35(0.57)$

Electrostatics short-range repulsive: $0.29(0.56)$ 
Electrostatics long-range attractive: 0.30 (1.09)

Electrostatics long-range repulsive: 0.21 (0.29)

ACE: 0.64 (0.7)

IFACE: $0.27(0.38)$

\section{Figures}

Plots were generated using gnuplot (www.gnuplot.info), and molecular visualizations were produced using PyMOL (www.pymol.org). Clustering and generation of the unrooted tree were performed using the APE package [37] in R (www.r-project.org).

\section{Additional files}

Additional file 1: Figure S1. Receptor interface RMSD versus ligand RMSD for test cases 4EI5 and 4LHU.

Additional file 2: Figure S2. Top-ranked ZRT model for test case 4EI5, showing (A) complex and (B) a chain interface with CD1d-Ag. CD1d is green, crystal structure TCR $a$ and $\beta$ chains are slate and tan, predicted $\mathrm{TCR}$ a and $\beta$ chains are orange and blue, unbound Ag is magenta, bound Ag is cyan, and predicted Ag is yellow.

Additional file 3: Table S1. Docking performance when initiated from the "start2" site.

Additional file 4: Figure S3. ZRT score versus interface RMSD for docking test cases. Red circles represent models from the "start1" docking start site, while blue triangles represent models from the "start2" docking start site.

Additional file 5: Figure S4. ZRT score versus interface RMSD for docking test case $4 \mathrm{LHU}$, using four docking start positions. Docking start sites shown are "start1" (red circles), "start2" (blue triangles), "start3" (magenta triangles), and "start4" (cyan circles).

Additional file 6: Figure S5. Distances between top 20 models (ranked by ZRT score) of Clone 18 GEM TCR (4G8E) bound to CD1b-MA and models of Clone 42 GEM TCR (4G8F) bound to CD1b-GMM, calculated using shared (identical in sequence) CDR1a and CDR2a loops. The lowest RMSD among all pairs of models $(1.37 \AA)$ is boxed, and corresponds to the predictions selected for further analysis (4G8E model 3 and 4G8F model 12).

Additional file 7: Figure S6. RMSD-based clustering of the top 20 $4 \mathrm{G} 8 \mathrm{E}$ and 4G8F models bound to CD1b-Ag. Selected sets of models are circled to indicate similarity with existing TCR complex crystal structures based on comparison of variable domain orientations after superposition of $\mathrm{MHC}$ or MHC-like structures.

Additional file 8: Figure S7. Additional GEM TCR-CD1b-Ag models compared with TCR-pMHC structures. Shown are (A) 4G8E-CD1b-MA model 18 and 1G4 TCR-HLA-A2-peptide (PDB code 2BNR), and (B) 4G8FCD1b-GMM model 5 and TRBV6 MAIT TCR-MR1-6FP CD1b-Ag (PDB code 4L4V). Colors are: GEM TCR a chains, blue; GEM TCR $\beta$ chains, salmon; crystallographic a chain, cyan; crystallographic $\beta$ chain, yellow; CD1b/ MR1/MHC, green; antigens, magenta.

Additional file 9: Figure S8. ZRT score versus interface RMSD for the 4L4T test case models (from the original "start1" docking position) evaluated against the recently released crystal structure of the same MAIT TCR in complex with a distinct Ag and MR1 (PDB code 4PJ8).

\section{Competing interests}

The authors declare that they have no competing interests.

\section{Authors' contributions}

BGP conceived the study, collected data, performed docking simulations, analyzed results, and wrote the manuscript. ZW helped to guide the study, and TV and ZW contributed to the writing of the manuscript. All authors read and approved the final manuscript.

\section{Acknowledgements}

We thank Mary Ellen Gipson-Fitzpatrick (Boston University) for computing support, and Lawrence Stern for valuable discussions.

\section{Funding}

This work was funded by NIH grant GM103773.

Received: 15 June 2014 Accepted: 22 September 2014

Published: 26 September 2014

\section{References}

1. Rudolph MG, Stanfield RL, Wilson IA: How TCRs bind MHCs, peptides, and coreceptors. Annu Rev Immunol 2006, 24:419-466.

2. Adams EJ: Lipid presentation by human CD1 molecules and the diverse T cell populations that respond to them. Curr Opin Immunol 2014, 26:1-6.

3. Kjer-Nielsen L, Patel O, Corbett AJ, Le Nours J, Meehan B, Liu L, Bhati M, Chen Z, Kostenko L, Reantragoon R, Williamson NA, Purcell AW, Dudek NL, McConville MJ, O'Hair RA, Khairallah GN, Godfrey DI, Fairlie DP, Rossjohn J, McCluskey J: MR1 presents microbial vitamin B metabolites to MAIT cells. Nature 2012, 491(7426):717-723.

4. Borg NA, Wun KS, Kjer-Nielsen L, Wilce MC, Pellicci DG, Koh R, Besra GS, Bharadwaj M, Godfrey DI, McCluskey J, Rossjohn J: CD1d-lipid-antigen recognition by the semi-invariant NKT T-cell receptor. Nature 2007 , 448(7149):44-49.

5. Patel O, Pellicci DG, Gras S, Sandoval-Romero ML, Uldrich AP, Mallevaey T, Clarke AJ, Le Nours J, Theodossis A, Cardell SL, Gapin L, Godfrey DI, Rossjohn J: Recognition of CD1d-sulfatide mediated by a type II natural killer T cell antigen receptor. Nat Immunol 2012, 13(9):857-863.

6. Girardi E, Maricic I, Wang J, Mac TT, Iyer P, Kumar V, Zajonc DM: Type II natural killer T cells use features of both innate-like and conventional T cells to recognize sulfatide self antigens. Nat Immuno/ 2012, 13(9):851-856.

7. Lopez-Sagaseta J, Dulberger CL, Crooks JE, Parks CD, Luoma AM, McFedries A, Van Rhijn I, Saghatelian A, Adams EJ: The molecular basis for MucosalAssociated Invariant T cell recognition of MR1 proteins. Proc Natl Acad Sci U S A 2013, 110(19):E1771-E1778.

8. Patel O, Kjer-Nielsen L, Le Nours J, Eckle SB, Birkinshaw R, Beddoe T, Corbett AJ, Liu L, Miles JJ, Meehan B, Reantragoon R, Sandoval-Romero ML, Sullivan LC, Brooks AG, Chen Z, Fairlie DP, McCluskey J, Rossjohn J: Recognition of vitamin B metabolites by mucosal-associated invariant T cells. Nat Commun 2013, 4:2142.

9. Uldrich AP, Le Nours J, Pellicci DG, Gherardin NA, McPherson KG, Lim RT, Patel O, Beddoe T, Gras S, Rossjohn J, Godfrey DI: CD1d-lipid antigen recognition by the gammadelta TCR. Nat Immunol 2013, 14(11):1137-1145

10. Luoma AM, Castro CD, Mayassi T, Bembinster LA, Bai L, Picard D, Anderson B, Scharf L, Kung JE, Sibener LV, Savage PB, Jabri B, Bendelac A, Adams EJ: Crystal structure of Vdelta1 $\mathrm{T}$ cell receptor in complex with CD1d-sulfatide shows MHC-like recognition of a self-lipid by human gammadelta T cells. Immunity 2013, 39(6):1032-1042.

11. Pierce $B G$, Weng $Z$ : $A$ flexible docking approach for prediction of $T$ cell receptor-peptide-MHC complexes. Protein Sci 2013, 22(1):35-46.

12. Berman HM, Westbrook J, Feng Z, Gilliland G, Bhat TN, Weissig H, Shindyalov IN, Bourne PE: The Protein Data Bank. Nucleic Acids Res 2000, 28(1):235-242

13. Eckle SB, Turner SJ, Rossjohn J, McCluskey J: Predisposed alphabeta T cell antigen receptor recognition of $\mathrm{MHC}$ and $\mathrm{MHC}-1$ like molecules? Curr Opin Immunol 2013, 25(5):653-659.

14. Lopez-Sagaseta J, Dulberger CL, McFedries A, Cushman M, Saghatelian A, Adams EJ: MAIT recognition of a stimulatory bacterial antigen bound to MR1. J Immunol 2013, 191(10):5268-5277.

15. Pellicci DG, Patel O, Kjer-Nielsen L, Pang SS, Sullivan LC, Kyparissoudis K, Brooks AG, Reid HH, Gras S, Lucet IS, Koh R, Smyth MJ, Mallevaey T, Matsuda JL, Gapin L, McCluskey J, Godfrey DI, Rossjohn J: Differential recognition of CD1d-alpha-galactosyl ceramide by the $\mathrm{V}$ beta 8.2 and $\mathrm{V}$ beta 7 semi-invariant NKT T cell receptors. Immunity 2009, 31(1):47-59.

16. Kjer-Nielsen L, Borg NA, Pellicci DG, Beddoe T, Kostenko L, Clements CS, Williamson NA, Smyth MJ, Besra GS, Reid HH, Bharadwaj M, Godfrey DI, Rossjohn J, McCluskey J: A structural basis for selection and cross-species reactivity of the semi-invariant NKT cell receptor in CD1d/glycolipid recognition. J Exp Med 2006, 203(3):661-673. 
17. Koch M, Stronge VS, Shepherd D, Gadola SD, Mathew B, Ritter G, Fersht AR, Besra GS, Schmidt RR, Jones EY, Cerundolo V: The crystal structure of human CD1d with and without alpha-galactosylceramide. Nat Immunol 2005, 6(8):819-826.

18. Zajonc DM, Maricic I, Wu D, Halder R, Roy K, Wong CH, Kumar V, Wilson IA: Structural basis for CD1d presentation of a sulfatide derived from myelin and its implications for autoimmunity. J Exp Med 2005, 202(11):1517-1526.

19. Reantragoon R, Kjer-Nielsen L, Patel O, Chen Z, Illing PT, Bhati M, Kostenko L, Bharadwaj M, Meehan B, Hansen TH, Godfrey DI, Rossjohn J, McCluskey J: Structural insight into MR1-mediated recognition of the mucosal associated invariant T cell receptor. J Exp Med 2012, 209(4):761-774.

20. Hwang H, Pierce B, Mintseris J, Janin J, Weng Z: Protein-protein docking benchmark version 3.0. Proteins 2008, 73(3):705-709.

21. Mendez R, Leplae R, De Maria L, Wodak SJ: Assessment of blind predictions of protein-protein interactions: current status of docking methods. Proteins 2003, 52(1):51-67.

22. Pierce B, Weng Z: A combination of rescoring and refinement significantly improves protein docking performance. Proteins 2008, 72(1):270-279.

23. Garcia KC, Degano M, Pease LR, Huang M, Peterson PA, Teyton L, Wilson IA: Structural basis of plasticity in $\mathrm{T}$ cell receptor recognition of a self peptide-MHC antigen. Science 1998, 279(5354):1166-1172.

24. Van Rhijn I, Kasmar A, de Jong A, Gras S, Bhati M, Doorenspleet ME, de Vries N, Godfrey DI, Altman JD, de Jager W, Rossjohn J, Moody DB: A conserved human $\mathrm{T}$ cell population targets mycobacterial antigens presented by CD1b. Nat Immunol 2013, 14(7):706-713.

25. Batuwangala T, Shepherd D, Gadola SD, Gibson KJ, Zaccai NR, Fersht AR Besra GS, Cerundolo V, Jones EY: The crystal structure of human CD1b with a bound bacterial glycolipid. J Immunol 2004, 172(4):2382-2388.

26. Adams EJ, Chien YH, Garcia KC: Structure of a gammadelta T cell receptor in complex with the nonclassical MHC T22. Science 2005, 308(5719):227-231.

27. Melian A, Watts GF, Shamshiev A, De Libero G, Clatworthy A, Vincent M, Brenner MB, Behar S, Niazi K, Modlin RL, Almo S, Ostrov D, Nathenson SG, Porcelli SA: Molecular recognition of human CD1b antigen complexes: evidence for a common pattern of interaction with alpha beta TCRs. $J$ Immunol 2000, 165(8):4494-4504.

28. Grant EP, Degano M, Rosat JP, Stenger S, Modlin RL, Wilson IA, Porcelli SA, Brenner MB: Molecular recognition of lipid antigens by $\mathrm{T}$ cell receptors. J Exp Med 1999, 189(1):195-205.

29. Eckle SB, Birkinshaw RW, Kostenko L, Corbett AJ, McWilliam HE, Reantragoon R, Chen Z, Gherardin NA, Beddoe T, Liu L, Patel O, Meehan B, Fairlie DP, Villadangos JA, Godfrey DI, Kjer-Nielsen L, McCluskey J, Rossjohn J: A molecular basis underpinning the T cell receptor heterogeneity of mucosal-associated invariant T cells. J Exp Med 2014, 211(8):1585-1600.

30. Kato Z, Stern JN, Nakamura HK, Kuwata K, Kondo N, Strominger JL: Positioning of autoimmune TCR-Ob.2 F3 and TCR-Ob.3D1 on the MBP8599/HLA-DR2 complex. Proc Natl Acad Sci U S A 2008, 105(40):15523-15528.

31. Leimgruber A, Ferber M, Irving M, Hussain-Kahn H, Wieckowski S, Derre L, Rufer $\mathrm{N}$, Zoete V, Michielin O: TCRep 3D: an automated in silico approach to study the structural properties of TCR repertoires. PLoS One 2011, 6(10):e26301.

32. Willcox CR, Pitard V, Netzer S, Couzi L, Salim M, Silberzahn T, Moreau JF, Hayday $A C$, Willcox BE, Dechanet-Merville J: Cytomegalovirus and tumor stress surveillance by binding of a human gammadelta $T$ cell antigen receptor to endothelial protein C receptor. Nat Immunol 2012, 13(9):872-879.

33. Eswar N, Webb B, Marti-Renom MA, Madhusudhan MS, Eramian D, Shen MY, Pieper U, Sali A: Comparative protein structure modeling using Modeller. Curr Protoc Bioinform 2006, Chapter 5:Unit 5-Unit 6.

34. O'Boyle NM, Banck M, James CA, Morley C, Vandermeersch T, Hutchison GR: Open Babel: an open chemical toolbox. J Cheminform 2011, 3:33.

35. Lemmon G, Meiler J: Rosetta Ligand docking with flexible XML protocols. Methods Mol Biol 2012, 819:143-155.

36. Pierce $B$, Weng Z: ZRANK: reranking protein docking predictions with an optimized energy function. Proteins 2007, 67(4):1078-1086.

37. Paradis E, Claude J, Strimmer K: APE: analyses of Phylogenetics and Evolution in R language. Bioinformatics 2004, 20(2):289-290.

doi:10.1186/1471-2105-15-319

Cite this article as: Pierce et al:: Modeling $T$ cell receptor recognition of CD1-lipid and MR1-metabolite complexes. BMC Bioinformatics 2014 15:319.

\section{Submit your next manuscript to BioMed Central and take full advantage of:}

- Convenient online submission

- Thorough peer review

- No space constraints or color figure charges

- Immediate publication on acceptance

- Inclusion in PubMed, CAS, Scopus and Google Scholar

- Research which is freely available for redistribution

Submit your manuscript at www.biomedcentral.com/submit
C BioMed Central 